\title{
Concordance of Acquired Mutations between Metastatic Lesions and Liquid Biopsy in Metastatic Colorectal Cancer Treated with Anti-EGFR Therapy
}

Fumitaka Taniguchi

Okayama University - Tsushima Campus: Okayama Daigaku

Akihiro Nyuya

Kawasaki Medical School: Kawasaki Ika Daigaku

Toshiaki Toshima

Okayama University: Okayama Daigaku

Yoshiko Mori

Saitama Ika Daigaku Sogo Iryo Center

Hiroyuki Kishimoto

Okayama University: Okayama Daigaku

Yuzo Umeda

Okayama University: Okayama Daigaku

\section{Toshiyoshi Fujiwara}

Okayama University: Okayama Daigaku

Takeshi Nagasaka ( $\nabla$ takeshin@cc.okayama-u.ac.jp)

Okayama University Graduate School of Medicine, Dentistry and Pharmaceutical Sciences

https://orcid.org/0000-0002-4236-350X

\section{Research article}

Keywords: metastatic colorectal cancer, acquired mutation, liquid biopsy, circulating cell-free tumor DNA, anti-EGFR treatment

Posted Date: October 13th, 2020

DOl: https://doi.org/10.21203/rs.3.rs-91128/v1

License: (c) (1) This work is licensed under a Creative Commons Attribution 4.0 International License.

Read Full License 


\section{Abstract}

Background: Acquired mutations are detected in circulating cell-free DNA or circulating tumor cells in blood. However, there are still few reports that examine the concordance between liquid biopsy and metastatic lesions with acquired resistance.

Methods: We examined the presence of acquired mutations in 7 chemoresistant metastatic lesions and blood samples obtained from a metastatic colorectal cancer ( $\mathrm{mCRC}$ ) patient without RAS activating mutations treated with anti-EGFR treatment. The patient displayed initial early tumor shrinkage and finally progressed to disease (PD). The 7 metastatic lesions showing acquired resistance to chemotherapy were extracted at morbid autopsy. Blood samples were collected before the development of PD during first-line chemotherapy and after acquiring resistance to second- and third-line chemotherapies. Acquired mutations were analyzed using two methodologies: conventional Sanger sequencing and MEBGENTM RASKET KIT based on Luminex ${ }^{\circledR}$ technology.

Results: Metastatic tumor specimens harbored diverse acquired mutations in the KRAS gene in all of the $7(100 \%)$ metastases in the patient resistant to EGFR-targeted treatment. Of the 7 diverse acquired mutations, only one acquired mutation observed in a liver metastasis was clearly detected in the blood collected at third-line chemotherapies. Two other acquired mutations were also detected but did not reach meaningful values.

Conclusions: Liquid biopsy is a successful procedure for capturing acquired mutations that may arise from progressive metastatic lesions with acquired resistance over time.

\section{Introduction}

Acquired resistance and primary resistance play major roles in anticancer treatment[1-3]. The epidermal growth factor receptor (EGFR)-targeted antibodies cetuximab and panitumumab are used to treat metastatic colorectal cancer (CRC) negative for mutations in KRAS and NRAS exons 2-4[4-6]. Although patients with metastatic CRC without activating RAS mutations generally show a clinical response to antiEGFR antibodies, acquired resistance may eventually develop. Several studies have identified acquired genetic alterations; e.g., KRAS, HER2, or MET amplification or KRAS, NRAS, BRAF, or EGFR mutations[1, 3, 7-12].

Recent studies have suggested that genomic alterations in solid tumors can be characterized by analyzing circulating tumor DNA (ctDNA), which is released from cancer cells into the plasma[13]. Currently, OncoBEAM-based liquid biopsy is a standard procedure for the detection of RAS mutations in plasma[14, 15]. Indeed, a previous study used OncoBEAM technology, which can detect mutant allele frequency (MAF) as low as $0.1 \%$ of acquired RAS mutant alleles $[1,16]$. However, even when using this procedure, approximately $10-18 \%$ of patients had $R A S$ mutations in tissue that could not be detected in plasma[14, 15]. 
The reason for this discordance may be attributed to tumor heterogeneity, lower circulating tumor DNA shedding or lower tumor burden. Clinically, mCRC patients at advanced stages possess multiple metastatic lesions in multiple organs. Therefore, in such cases, heterogeneity of $R A S$ mutations must be considered. However, there are few reports evaluating the concordance of RAS mutational status in all metastatic lesions located in multiple organs and ctDNA.

In this study, we used PCR-rSSO, which can detect an MAF of $1-5 \%$ of the RAS mutant allele, and is in concordance with the detection of RAS mutations with reports of $93.9 \%$ using a $5 \%$ cut-off of tissue. OncoBEAMing was used and examined the concordance of RAS mutations observed between multiple metastatic lesions in multiple organs and ctDNA in plasma throughout anti-EGFR therapy in an mCRC patient[15].

\section{Methods}

\section{Tumor and Blood Samples}

mCRC patients with primary tumors without activating RAS mutations were enrolled in the study. The patients were treated between 2011 and 2015 at the Okayama University Hospital, Japan. This study was approved by the Institutional Review Board (IRB) at Okayama University (IRB number, genome 270 and 271) and Kawasaki Medical School (IRB number, 3196-1 and 3239). The patients provided written informed consent.

Primary tumors were obtained by biopsy and surgical resection before acquiring resistance during antiEGFR treatment. Metastatic lesions were excised at morbid autopsy and included those of the liver (MS, S2, and S3), lung (Lu), and kidney (Kd). Tissues obtained from autopsy or surgical resection were immediately stored at $-80^{\circ} \mathrm{C}$.

Blood samples were collected before developing PD during first-line chemotherapy (*20) and after acquiring resistance to second- and third-line chemotherapies ( $* 105$ and $* 123$, respectively; Fig. 1 ). Plasma was separated immediately and stored at $-80^{\circ} \mathrm{C}$.

\section{Extraction of Genomic DNA}

Genomic DNA was extracted from fresh frozen samples using the QIAamp DNA Mini Kit (Qiagen NV, Hilden, Netherlands). Tumor DNA of several metastatic lesions was microscopically extracted from formalin-fixed paraffin-embedded (FFPE) specimens. These specimens included metastases located in the liver (RL) and hepatic lymph node (HN). DNA derived from FFPE specimens was extracted using the QIAamp DNA FFPE Tissue Kit (Qiagen NV, Hilden, Netherlands). Circulating cell-free DNA was extracted from $200 \mu \mathrm{l}$ of plasma using the QIAamp Blood Kit (Qiagen) according to the manufacturer's instructions.

\section{Conventional Sequencing}

Sanger sequencing was also performed to confirm mutations in KRAS, NRAS exons 2 to 4 , and BRAF exon 15 (including codon 600) in the discovery cohort. Primary tumors from 1,070 CRC patients were 
analyzed to identify mutations in KRAS exon 2 and BRAF exon 15. Primer sequences for KRAS, NRAS exons 2 to 4, and BRAF exon 15 and PCR conditions are described in Supplementary File 1. PCR products were purified using the QIAquick PCR Purification Kit (Qiagen) and were directly sequenced using the ABI PRISM ${ }^{\circledR}$ 3100-Avant (Applied Biosystems) and a SeqStudio Genetic Analyzer (Thermo Fisher Scientific).

\section{Polymerase Chain Reaction-Reverse Sequence Specific Oligonucleotide (PCR-rSSO)}

Extended RAS mutations (both KRAS and NRAS mutations) of DNA purified from FFPE, fresh frozen tissues, and plasma were evaluated using the MEBGEN ${ }^{\mathrm{TM}}$ RASKET KIT based on Luminex ${ }^{\circledR}$ technology (MBL, Nagoya, Japan). The assay was performed according to the manufacturer's protocol. We simultaneously examined 12 types of RAS exon 2 (G12S, G12C, G12R, G12D, G12V, G12A, G13S, G13C, G13R, G13D, G13V, and G13A), eight types of RAS exon 3 (A59T, A59G, Q61K, Q61E, Q61L, Q61P, Q61R, and $\mathrm{Q} 61 \mathrm{H})$ and four types of $R A S$ exon $4(\mathrm{~K} 117 \mathrm{~N}, \mathrm{~A} 146 \mathrm{~T}, \mathrm{~A} 146 \mathrm{P}$, and $\mathrm{A} 146 \mathrm{~V})$ mutations[16].

\section{Results}

\section{Clinical Outcome}

One patient had multiple unresectable liver-limited metastases at their first medical examination and was treated with first-line systemic chemotherapy with anti-EGFR therapy (Fig. 1). The primary tumor had no mutations in KRAS, NRAS or BRAF exon 15 (Fig. 2). Overall survival after first-line treatment initiation was 34 months. The patient was administered bevacizumab with cytotoxic agents as the second-line regimen and was rechallenged with anti-EGFR antibody as the third-line chemotherapy. Despite initial tumor shrinkage in the metastases, the patient ultimately developed PD.

\section{Identification of Acquired RAS Mutations by Conventional Sanger Sequencing}

In the patient, no activating RAS mutation was observed in the primary tumor biopsy specimen obtained prior to treatment, in primary tumor tissue obtained after 1 year of panitumumab administration, or in a metastatic tumor in the right liver lobe (RL) that responded continuously to panitumumab with first-line systemic chemotherapy and shrank in response to third-line panitumumab rechallenge (Fig. 2). In addition, KRAS sequences in metastatic specimens obtained during autopsy revealed diverse acquired mutations at different metastatic sites, indicating resistance to the systemic chemotherapy used, including anti-EGFR antibody treatment. Acquired activating $K R A S$ mutations resulting in $\mathrm{G} 61 \mathrm{Hc}, \mathrm{G} 12 \mathrm{R}$, and G12V were detected by Sanger sequencing in liver segments II (S2), III (S3), and middle segment (MS), respectively. The KRASG12C, G13D, and G61 Hc mutations were also detected in metastases in the left kidney $(\mathrm{Kd})$, in a hepatic lymph node $(\mathrm{HN})$, and in the left lung $(\mathrm{Lu})$, respectively. No samples from primary and metastatic lesions harbored mutations in NRAS or BRAF.

\section{Identification of Acquired RAS Mutations by a PCR-rSSO Method}

By Sanger sequencing, acquired mutations were detected in $6(87 \%)$ of the 7 metastatic lesions. Because of the lower sensitivity for the detection of mutant alleles by Sanger sequencing ( 20\%), we could not 
exclude the possibility of associated metastatic lesions caused by resistant cells already existing in the primary lesion in extremely low numbers.

We further analyzed all samples using the PCR-rSSO method, RASKET, which has high sensitivity in terms of detecting extended $R A S$ mutant alleles at lower frequencies, 1-5\%; a sample was considered mutationpositive when the index was estimated over the cut-off value, the sensitivity of which for the mutant allele was $1-5 \%$, for each mutant allele[16].

Using this procedure, KRAS or NRAS mutations with indices higher than the cut-off values were not found in DNA purified from pretreated primary tumor cells (Fig. 3 and Supplementary File 2). Additionally, PCRrSSO analysis revealed the same mutation spectrum obtained by Sanger sequencing in the patient except for the metastatic lesion in the $\mathrm{Kd}$ and the $\mathrm{RL}$. The index of the KRASG12C allele captured from the $\mathrm{Kd}$ by PCR-rSSO analysis was 114, i.e., lower than the conventional cut-off value (300), which might be influenced by the immediate synonymous mutation of A11Ag clearly demonstrated by Sanger sequencing, as previously reported (Fig. 2) [16]. Interestingly, the metastatic tumor in the RL, which responded consistently to panitumumab and showed no mutation by Sanger sequencing, revealed multiple KRAS mutations, Q61 Ht, G12A, and G12R, with indices higher than the cut-off values. Although these mutant alleles were not frequent enough to be detected by Sanger sequencing, heterogeneity in RAS mutant cancer cells may exist in the RL tumor mass.

In summary, by Sanger sequencing and PCR-rSSO, the 7 metastatic lesions were found to harbored diverse acquired mutations in the KRAS gene: Q61Ht, G12A, and G12R in RL; G12V in MS; Q61 Hc in S2; G12R in S3; G12C in Kd; G13D in HN; Q61Hc in Lu.

\section{Detection of Acquired Mutations in Circulating Cell-Free DNA}

We investigated whether acquired RAS mutations could be detected in the plasma samples using PCRrSSO. The 7 diverse acquired mutations found in the resistant tumors were not detected before or after PD during the initial course of panitumumab treatment (*20 and *105 in Fig. 4 and Supplementary File 3), but only one of the 7 diverse acquired mutations was detected at a significant level following the

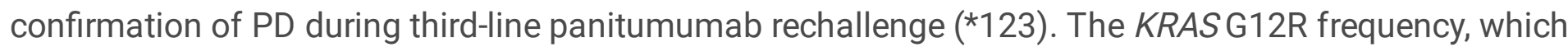
was confirmed in the resistant tumor in S3, was strikingly elevated in circulating cell-free DNA in a plasma sample collected 123 weeks after treatment initiation. Additionally, KRASG13D in the HN and KRAS Q61 Hc in the Lu and S2 were also detected in the plasma sample ${ }^{*} 123$, but the indices were not reached at the cut-off value. All the acquired mutations detected in the plasma sample *123 were not detected in plasma at 20 weeks ( $* 20$, approximately as early as 10 months before PD during first-line therapy) or at 105 weeks ( ${ }^{*} 105$, when PD was confirmed radiologically during first-line treatment).

\section{Discussion}

PD is generally determined by radiological evaluation. Liquid biopsy has been suggested for the early identification of individuals at risk of developing drug resistance prior to radiographic documentation of PD as well as individuals who already have possessed certain mutations in tumor burden[1, 13-15]. Here, 
PCR-rSSO analysis was conducted on serial plasma samples and all metastatic lesions in multiple organs from a patient who was treated with first- and third-line chemotherapy, including panitumumab, until PD.

In a previous study, an acquired mutation was detected in plasma as early as 10 months before radiological PD[1]; therefore, we attempted to confirm the detection of acquired RAS variants in plasma that were identified in metastatic tumor lesions. However, none of the 7 acquired mutations found in the resistant tumors were detected either before or after PD during the initial panitumumab treatment course (*20 and *105), but three of the 7 acquired mutations were detectable after PD was confirmed during third-line panitumumab rechallenge (*123). Indeed, the two of three acquired RAS mutations confirmed in the PCR-rSSO were under the cut-off values estimated by tumor tissue analysis. Our inability to detect ctDNA in the plasma samples might be explained partially by a difference in analytical technologies. The PCR-rSSO method used in the present study could detect MAFs of $1-5 \%$, whereas the previous study with OncoBEAMing technology considered $0.1 \%$ MAFs as mutation positive $[1,16]$. However, OncoBEAMing missed the detection of RAS mutations in plasma, and approximately $10-18 \%$ of patients had $R A S$ mutations in tumor tissue[14, 15], owing to tumor heterogeneity, lower ctDNA shedding or lower tumor burden. In particular, the location of metastatic tumors may be important for the detection of mutant alleles. Discrepancies of mutations between tumor tissue and ctDNA in patients with lung-only metastases were observed, but a higher agreement in liver metastases was found $[15,17,18]$. In line with this, our results also showed that the degree of $R A S$ mutational concordance varied according to the metastatic site; e.g., KRAS G12R clearly detected in ctDNA was confirmed in resistant tumors in $\mathrm{S} 3$, looking like more aggressively progressed by radiographic findings.

At least two possibilities account for the development of acquired mutations $[3,9]$. First, resistant cells harboring these acquired mutations may be present at very low numbers upon treatment initiation (Fig. 5a). Second, in response to continued molecular-targeted therapy-based chemotherapy, cells may have acquired a de novo activating mutation (Fig. $5 \mathrm{~b}$ ). In the first model, metastatic CRC response to EGFR-targeted therapies is accompanied by a selection of preexisting resistant clones metastasizing to the initial metastatic lesion; therefore, if the acquired RAS mutations observed in our case were present at treatment initiation but at a frequency sufficiently low to be undetectable by the two different procedures, at least some resistant metastatic lesions that grew after treatment should harbor not one but multiple $R A S$ mutations. However, almost all resistant metastatic lesions harbored one acquired RAS mutation, as demonstrated by conventional Sanger sequencing and the highly sensitive PCR-SSO procedure, except for RL, which displayed multiple KRAS mutants by PCR-rSSO with a lower population and was not detected by Sanger sequencing, suggesting the existence of many types of cancer cells. The lower population of multiple KRAS mutants could explain why the RL visibly continued shrinking with calcification during the sequential chemotherapy treatment, as visualized by computed tomography.zA

Since only a small part of the tumor lesions could be examined, we cannot exclude sampling bias with regard to the existence of mutant alleles precluding assessment of genetic heterogeneity within or among lesions. However, with respect to RAS mutations in CRC, RAS mutations are believed to occur in the early 
phase of tumorigenesis, such as in the development of a small adenoma into a larger adenoma[19]. Thus, an RAS mutation spreads homogenously within the tumor mass, resulting in more than $95 \%$ concordance in RAS mutation status across different sites of a tumor mass[20]. Therefore, RAS mutationbased drug resistance may be attributable to new mutations arising rather than the selection and clonal amplification of a very small number of preexisting RAS mutant cells. This scenario was also supported by previously reported in vitro studies[1, 21]. Shaffer SM et al. presented that in a BRAF-mutant melanoma cell line, the population of resistant cells may arise upon the selection of multiple clones that were already present before $B R A F$ inhibitor treatment[22]. Of note, these resistant cells arise from profound transcriptional variability at the single-cell level, which involves infrequent, semicoordinated transcription of a number of resistance markers at high levels in a very small percentage of cells. The addition of the drug then induced epigenetic reprogramming in these cells, converting the transient transcriptional state to a stably resistant state.

In this study, although we analyzed only one case and were unable to identify acquired mutations via liquid biopsy before radiographic documentation of $\mathrm{PD}$, we clearly detected acquired drug resistanceinducing mutations in liquid biopsy collected during a later phase of the treatment course, thus strongly emphasizing the clinical utility of liquid biopsy for decisions regarding anti-EGFR antibody administration to individuals at risk of drug resistance.

\section{Conclusions}

Our results indicate that anti-EGFR treatment induces the development of resistant cancer cells with acquired resistance heterogenetic mutations. At the time that a certain acquired mutation was detected by "liquid biopsy", it might strongly suggest that tumor cells with such acquired mutations definitely exist in progressive metastatic lesions.

\section{Declarations}

\section{Authors' contributions}

FT performed genetic analyses and drafted the manuscript. AN, TT, and KY extracted DNA and analyzed the genetic mutations. YM, HK, YU, and TF treated patients, provided the patient samples, and summarized all clinicopathological data. TN designed the project, performed analyses, assisted with the interpretation of all data, secured the funding, and drafted the manuscript. All authors read and approved the final manuscript.

\section{Grant Support}

This work was supported by KAKENHI $(20590572,25860409,26462016$, and 15H03034).

\section{Acknowledgments}


The authors would like to thank Dr. Futoshi Uno, Dr. Ayako Watanabe, Mr. Toru Nakai, Mrs. Tae Yamanishi, and Mrs. Kikue Tokuda for their technical assistance.

\section{References}

1. Misale S, Yaeger R, Hobor S, Scala E, Janakiraman M, Liska D, Valtorta E, Schiavo R, Buscarino M, Siravegna $\mathrm{G}$ et al: Emergence of KRAS mutations and acquired resistance to anti-EGFR therapy in colorectal cancer. Nature 2012, 486(7404):532-536.

2. Kobayashi S, Boggon TJ, Dayaram T, Janne PA, Kocher O, Meyerson M, Johnson BE, Eck MJ, Tenen DG, Halmos B: EGFR mutation and resistance of non-small-cell lung cancer to gefitinib. The New England journal of medicine 2005, 352(8):786-792.

3. Misale S, Di Nicolantonio F, Sartore-Bianchi A, Siena S, Bardelli A: Resistance to anti-EGFR therapy in colorectal cancer: from heterogeneity to convergent evolution. Cancer discovery 2014, 4(11):12691280.

4. Douillard JY, Oliner KS, Siena S, Tabernero J, Burkes R, Barugel M, Humblet Y, Bodoky G, Cunningham $\mathrm{D}$, Jassem $\mathrm{J}$ et al: Panitumumab-FOLFOX4 treatment and RAS mutations in colorectal cancer. The New England journal of medicine 2013, 369(11):1023-1034.

5. Heinemann V, von Weikersthal LF, Decker T, Kiani A, Vehling-Kaiser U, Al-Batran SE, Heintges T, Lerchenmuller C, Kahl C, Seipelt $\mathrm{G}$ et al: FOLFIRI plus cetuximab versus FOLFIRI plus bevacizumab as first-line treatment for patients with metastatic colorectal cancer (FIRE-3): a randomised, openlabel, phase 3 trial. The Lancet Oncology 2014, 15(10):1065-1075.

6. Van Cutsem E, Lenz HJ, Kohne CH, Heinemann V, Tejpar S, Melezinek I, Beier F, Stroh C, Rougier P, van Krieken JH et al: Fluorouracil, leucovorin, and irinotecan plus cetuximab treatment and RAS mutations in colorectal cancer. Journal of clinical oncology : official journal of the American Society of Clinical Oncology 2015, 33(7):692-700.

7. Yonesaka K, Zejnullahu K, Okamoto I, Satoh T, Cappuzzo F, Souglakos J, Ercan D, Rogers A, Roncalli $\mathrm{M}$, Takeda $\mathrm{M}$ et al: Activation of ERBB2 signaling causes resistance to the EGFR-directed therapeutic antibody cetuximab. Science translational medicine 2011, 3(99):99ra86.

8. Montagut C, Dalmases A, Bellosillo B, Crespo M, Pairet S, Iglesias M, Salido M, Gallen M, Marsters S, Tsai SP et al: Identification of a mutation in the extracellular domain of the Epidermal Growth Factor Receptor conferring cetuximab resistance in colorectal cancer. Nature medicine 2012, 18(2):221-223.

9. Diaz LA, Jr., Williams RT, Wu J, Kinde I, Hecht JR, Berlin J, Allen B, Bozic I, Reiter JG, Nowak MA et al: The molecular evolution of acquired resistance to targeted EGFR blockade in colorectal cancers. Nature 2012, 486(7404):537-540.

10. Bardelli A, Corso S, Bertotti A, Hobor S, Valtorta E, Siravegna G, Sartore-Bianchi A, Scala E, Cassingena $A$, Zecchin D et al: Amplification of the MET receptor drives resistance to anti-EGFR therapies in colorectal cancer. Cancer discovery 2013, 3(6):658-673. 
11. Bertotti A, Papp E, Jones S, Adleff V, Anagnostou V, Lupo B, Sausen M, Phallen J, Hruban CA, Tokheim $\mathrm{C}$ et al: The genomic landscape of response to EGFR blockade in colorectal cancer. Nature 2015.

12. Siravegna G, Mussolin B, Buscarino M, Corti G, Cassingena A, Crisafulli G, Ponzetti A, Cremolini C, Amatu A, Lauricella $\mathrm{C}$ et al: Clonal evolution and resistance to EGFR blockade in the blood of colorectal cancer patients. Nature medicine 2015, 21(7):795-801.

13. Bettegowda C, Sausen M, Leary RJ, Kinde I, Wang Y, Agrawal N, Bartlett BR, Wang H, Luber B, Alani $\mathrm{RM}$ et al: Detection of circulating tumor DNA in early- and late-stage human malignancies. Science translational medicine 2014, 6(224):224ra224.

14. Garcia-Foncillas J, Tabernero J, Elez E, Aranda E, Benavides M, Camps C, Jantus-Lewintre E, Lopez R, Muinelo-Romay L, Montagut $\mathrm{C}$ et al: Prospective multicenter real-world RAS mutation comparison between OncoBEAM-based liquid biopsy and tissue analysis in metastatic colorectal cancer. $\mathrm{Br} \mathrm{J}$ Cancer 2018, 119(12):1464-1470.

15. Bando H, Kagawa Y, Kato T, Akagi K, Denda T, Nishina T, Komatsu Y, Oki E, Kudo T, Kumamoto H et al: A multicentre, prospective study of plasma circulating tumour DNA test for detecting RAS mutation in patients with metastatic colorectal cancer. Br J Cancer 2019, 120(10):982-986.

16. Yoshino T, Muro K, Yamaguchi K, Nishina T, Denda T, Kudo T, Okamoto W, Taniguchi H, Akagi K, Kajiwara T et al: Clinical Validation of a Multiplex Kit for RAS Mutations in Colorectal Cancer: Results of the RASKET (RAS KEy Testing) Prospective, Multicenter Study. EBioMedicine 2015, 2(4):317-323.

17. Kim MJ, Lee HS, Kim JH, Kim YJ, Kwon JH, Lee JO, Bang SM, Park KU, Kim DW, Kang SB et al: Different metastatic pattern according to the KRAS mutational status and site-specific discordance of KRAS status in patients with colorectal cancer. BMC Cancer 2012, 12:347.

18. Thierry AR, El Messaoudi S, Mollevi C, Raoul JL, Guimbaud R, Pezet D, Artru P, Assenat E, Borg C, Mathonnet $\mathrm{M}$ et al: Clinical utility of circulating DNA analysis for rapid detection of actionable mutations to select metastatic colorectal patients for anti-EGFR treatment. Ann Oncol 2017, 28(9):2149-2159.

19. Jones S, Chen WD, Parmigiani G, Diehl F, Beerenwinkel N, Antal T, Traulsen A, Nowak MA, Siegel C, Velculescu VE et al: Comparative lesion sequencing provides insights into tumor evolution. Proceedings of the National Academy of Sciences of the United States of America 2008, 105(11):4283-4288.

20. Krol LC, t Hart NA, Methorst N, Knol AJ, Prinsen C, Boers JE: Concordance in KRAS and BRAF mutations in endoscopic biopsy samples and resection specimens of colorectal adenocarcinoma. European journal of cancer 2012, 48(7):1108-1115.

21. Misale S, Arena S, Lamba S, Siravegna G, Lallo A, Hobor S, Russo M, Buscarino M, Lazzari L, SartoreBianchi $A$ et al: Blockade of EGFR and MEK intercepts heterogeneous mechanisms of acquired resistance to anti-EGFR therapies in colorectal cancer. Science translational medicine 2014, 6(224):224ra226. 
22. Shaffer SM, Dunagin MC, Torborg SR, Torre EA, Emert B, Krepler C, Beqiri M, Sproesser K, Brafford PA, Xiao $\mathrm{M}$ et al: Rare cell variability and drug-induced reprogramming as a mode of cancer drug resistance. Nature 2017, 546(7658):431-435.

\section{Figures}

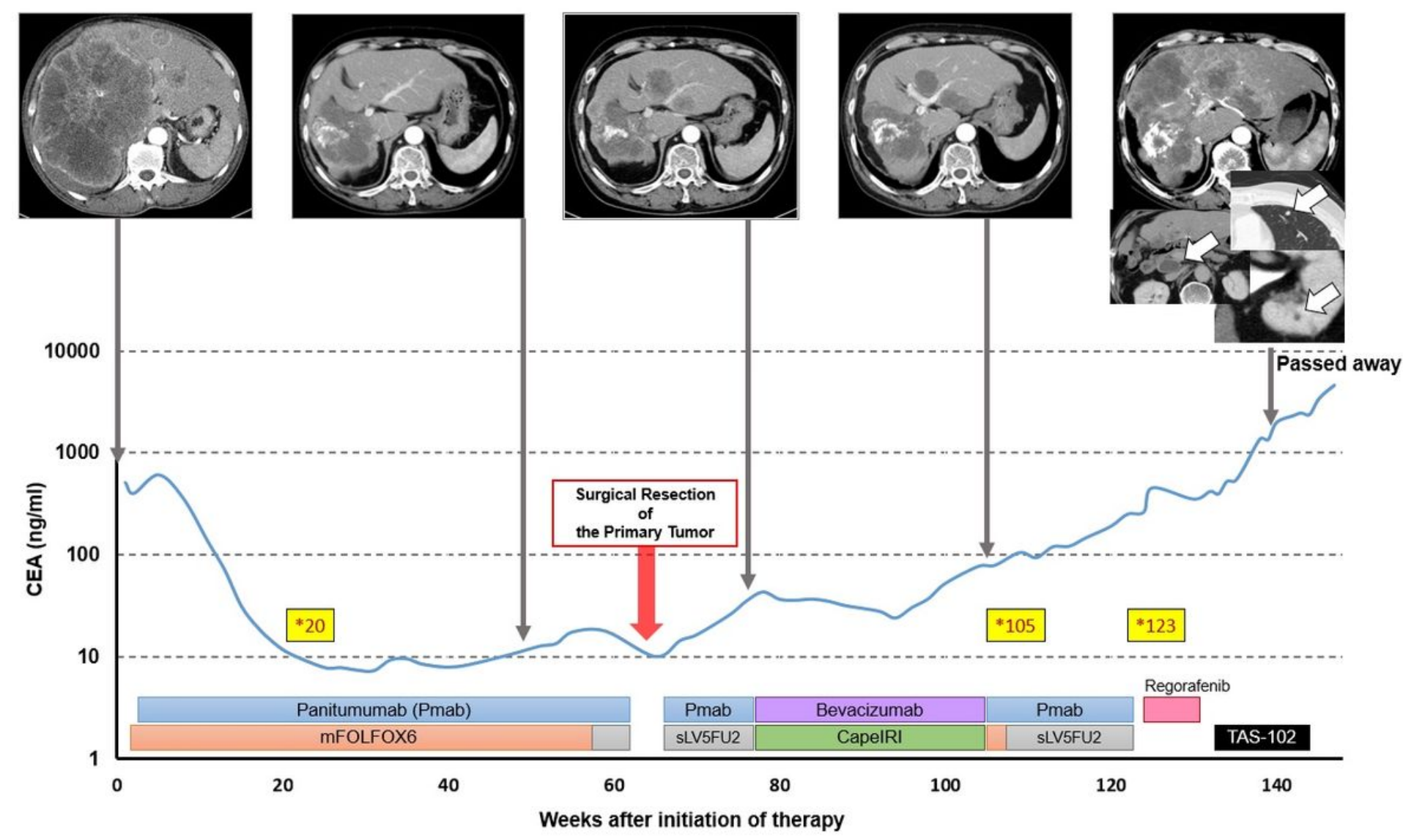

\section{Figure 1}

Timeline of a rectosigmoid cancer patient with unresectable multiple liver metastases. The line graph abscissa indicates time, and the ordinate indicates carcinoembryonic CEA levels, scaled logarithmically. The computed tomography scan of the liver metastases (above) and chemotherapy regimen (below) are described for each time point. Blood was collected before the administration of chemotherapy at 20 (*20), 105 (*105), and 123 (*123) weeks after the initiation of first-line chemotherapy. White arrows denote metastatic lesions in the hepatic lymph node, the left lung, and the left kidney. 


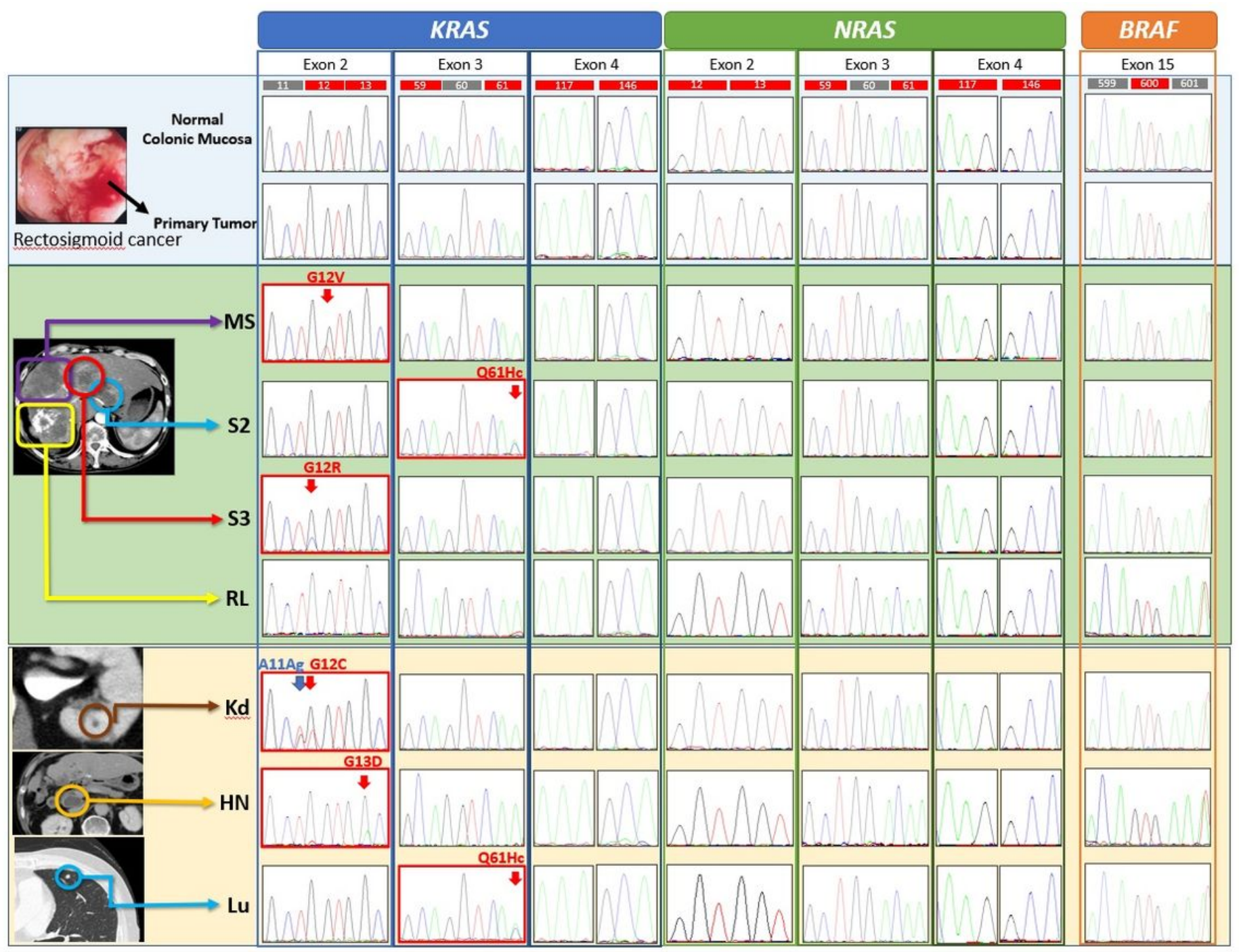

Figure 2

Status of RAS and BRAF Mutational Metastases by Sanger Sequencing The status of KRAS, NRAS and BRAF. Sanger sequencing results are described for all samples, including primary lesions, with focal distant metastasis. Numbers in red and gray boxes indicate the codon numbers. Codons in red boxes indicate hot spots. Red arrows indicate observed missense mutations. 

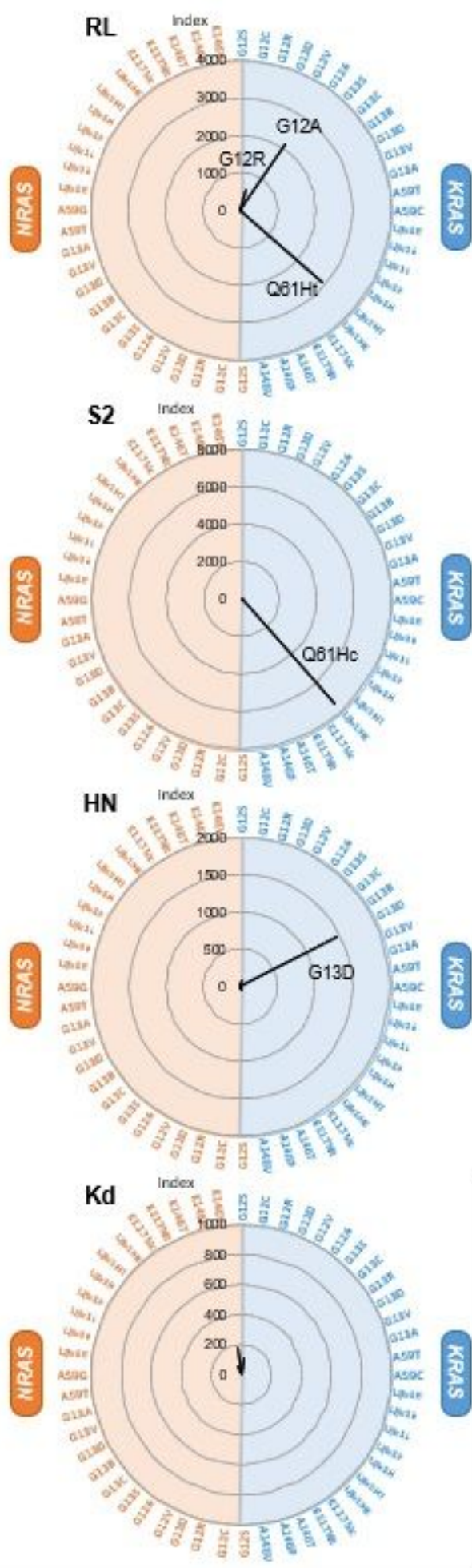

MS

圆

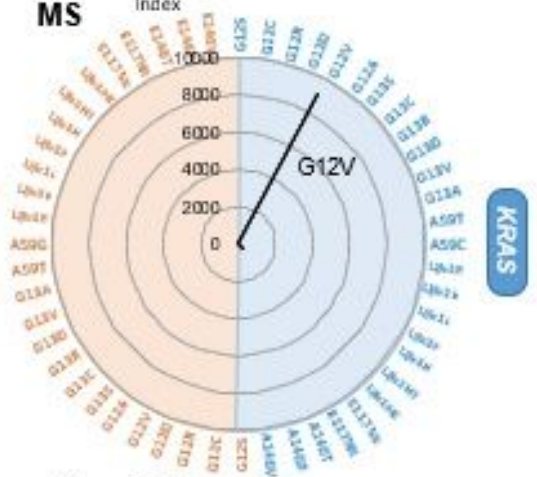

S3

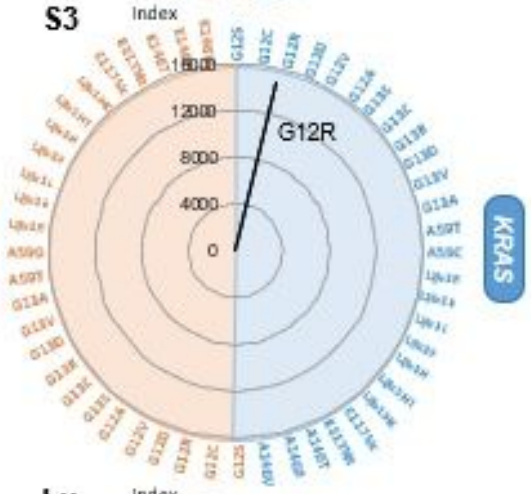

Lu Index
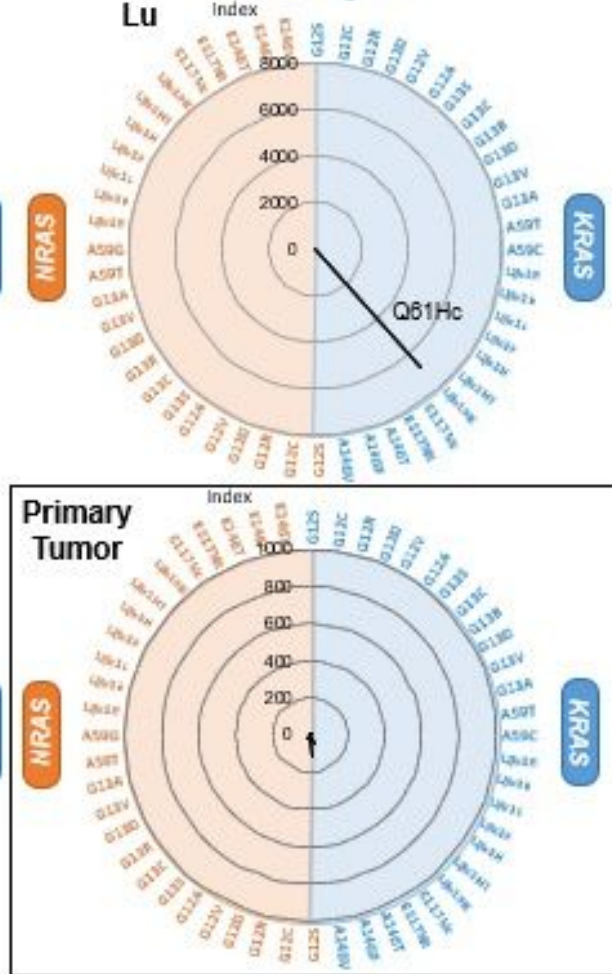

\section{Figure 3}

Status of RAS Mutational Metastases by PCR-rSSO. Panels show the radar charts, indicating PCR-rSSO indices of metastatic specimens in the patient. The right-sided light blue semicircle shows KRAS mutations, and the left-sided light orange semicircle shows NRAS mutations. 


\section{KRAS codon 12}

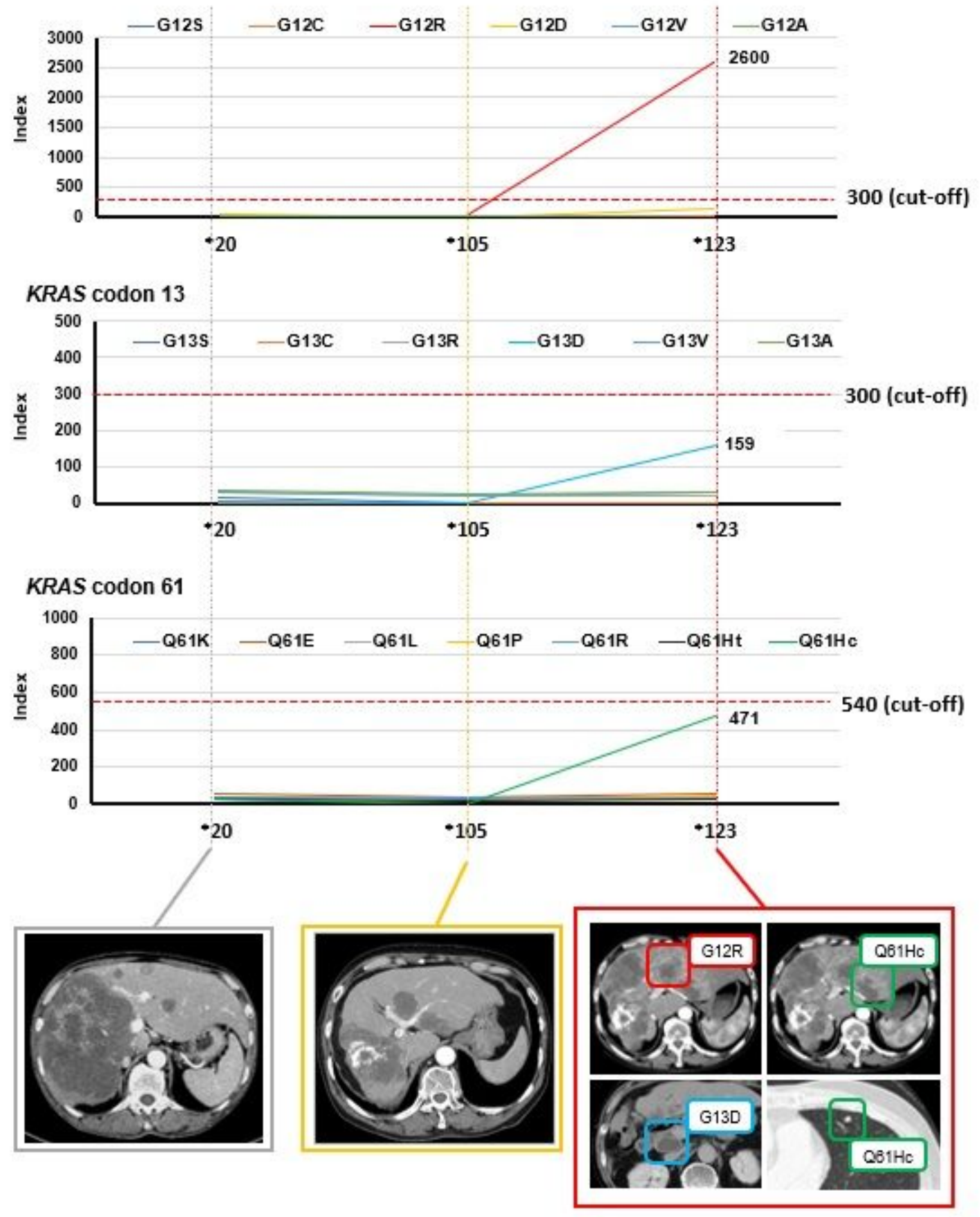

\section{Figure 4}

Detection of Circulating KRAS Mutant DNA The detection of circulating KRAS mutant alleles in plasma by PCR-rSSO. The KRAS G12R mutant allele index value increased to 2,600 in plasma obtained at 123 weeks (*123) after the initiation of the treatment. The index values of the KRAS G13D and Q61 Hc mutant alleles also increased to 159 and 471, respectively, in plasma obtained at 123 weeks (*123). 
a

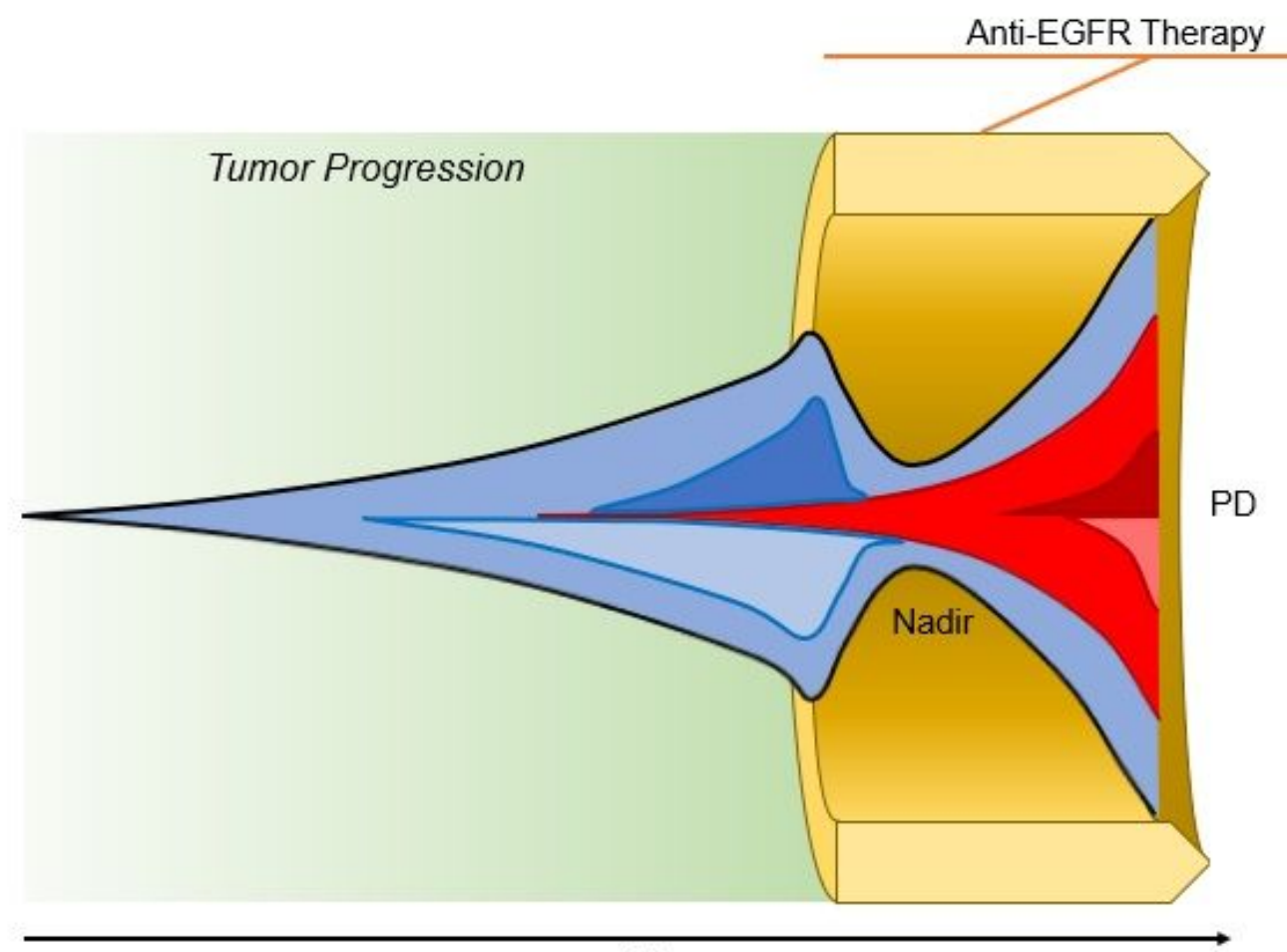

Time

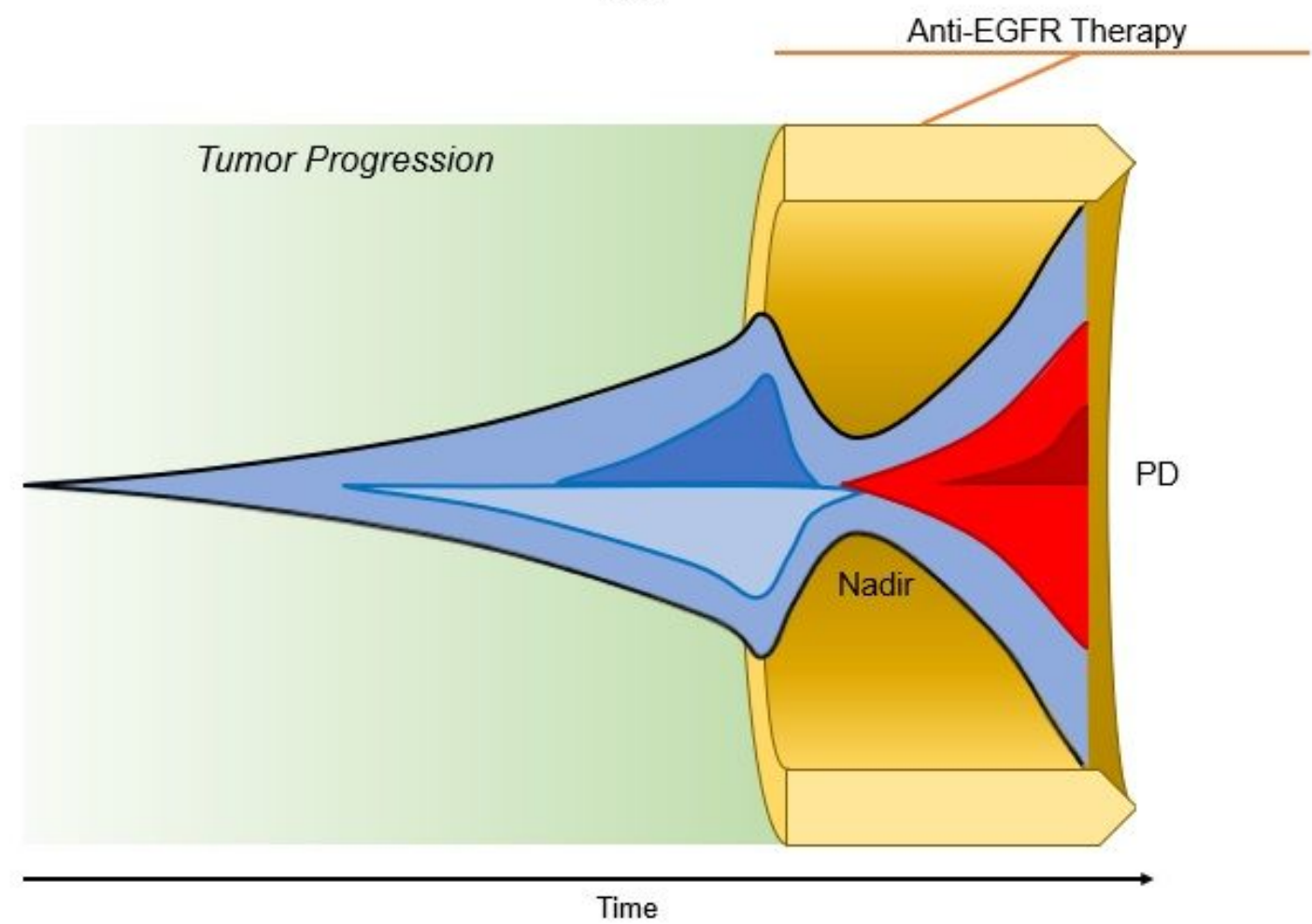

\section{Figure 5}

Possible mechanisms of acquired resistance to anti-EGFR therapies in metastatic CRC. (a) A possible mechanism of acquired resistance caused by the already existing heterogeneity and resistant cancer cells carrying distinct molecular alterations, such as KRAS (red), coexist in the initial metastasis and/or primary tumor sites. (b) Another possible mechanism of acquired resistance caused by a cell, which acquires a de novo activating acquired mutation in response to continued EGFR-targeted therapy-based 
chemotherapy. Cancer cells that acquire a de novo activating acquired RAS mutation appear after EGFRtargeted therapy-based chemotherapy (red).

\section{Supplementary Files}

This is a list of supplementary files associated with this preprint. Click to download.

- TaniguchiFetal.SupplementaryFile1.xlsx

- TaniguchiFetal.SupplementaryFile2.xlsx

- TaniguchiFetal.SupplementaryFile3.xlsx 